\title{
Prelimbic proBDNF facilitates memory destabilization by regulation of neuronal function in juveniles
}

\author{
Wei Sun ${ }^{1,2}$, Xiao Chen ${ }^{2}$, Yazi Mei ${ }^{3}$, Yang Yang ${ }^{1}$, Xiaoliang $\mathrm{Li}^{4}$, Lei $\mathrm{An}^{1,2 *}$ \\ ${ }^{1}$ Department of Pediatric, The First Affiliated Hospital, Guizhou University of \\ Traditional Chinese Medicine, Guiyang, Guizhou 550001, China; \\ ${ }^{2}$ Behavioral Neuroscience Laboratory, The First Affiliated Hospital, Guizhou \\ University of Traditional Chinese Medicine, Guiyang, Guizhou 550001, China; \\ ${ }^{3}$ Graduate School of Guangzhou University of Chinese Medicine; Guangzhou 510006, \\ China; \\ ${ }^{4}$ Department of Neurology, Jinan Geriatric/Rehabilitation Hospital, Jinan 250013, \\ China
}

Running title: Prefrontal proBDNF and fear memory

Tel/Fax: +86-20-39356092

*Correspondence to LA (al_totti@sina.com; leian.nku@gmail.com;

anlei776@gzy.edu.cn; lei.an@gzucm.edu.cn) 


\begin{abstract}
Fear regulation changes as a function of age and adolescence is a key developmental period for the continued maturation of fear neural circuitry. The involvement of prelimbic proBDNF in fear memory extinction and its mediated signaling were reported previously. Given the inherent high level of proBDNF during juvenile period, we tested whether prelimbic proBDNF regulated synaptic and neuronal functions allowing to influencing retrieval-dependent memory processing. By examining freezing behavior of auditory fear conditioned rats, we found high levels of prelimbic proBDNF in juvenile rats enhanced destabilization of the retrieval-dependent weak but not strong fear memory through activating p75 ${ }^{\mathrm{NTR}}$-GluN2B signaling. This modification was attributed to the increment in proportion of thin type spine and promotion in synaptic function, as evidence by facilitation of NMDA-mediated EPSCs and GluN2B-dependent synaptic depression. The strong prelimbic theta- and gamma-oscillation coupling predicted the suppressive effect of proBDNF on the recall of post-retrieval memory. Our results critically emphasize the importance of developmental proBDNF for modification of retrieval-dependent memory and provide a potential critical targeting to inhibit threaten memories associated with neurodevelopment disorders.
\end{abstract}

Keywords: memory retrieval; neural correlate; prelimbic cortex; proBDNF; synaptic plasticity 


\section{Introduction}

Fear can be highly adaptive in promoting survival, yet it can also be detrimental when it persists long after a threat has passed (Kim-Cohen et al, 2003; Liberman et al, 2006; Moriceau and Sullivan, 2006). Flexibility of the fear response may be most advantageous during the early development period when animals are prone to explore novel, potentially threatening environments (Kim et al, 2011; McCallum et al, 2010; Pattwell et al, 2012). Actually, dynamic changes in neural circuitry during the sensitive period of neural development can induce memory to enter a destabilized state and attenuate maladaptive memories associated with emotional disorders (Pattwell et al, 2011; Pattwell et al, 2016). Retrieval of an existing fear memory can trigger either maintenance or inhibition of the stored memories depending on the degree of re-exposure to fear cues (Merlo et al, 2018). These opposing fear-related behaviors depend on distinct components of a medial prefrontal cortical circuit that receive projections from the amygdala and ventral hippocampus (Milad and Quirk, 2002; Sotres-Bayon et al, 2012). Further researches indicate that the developmental changes in the extinction and recall of cued fear memories involve protracted development of prelimbic region of medial prefrontal cortex (mPFC) (Da Silva et al, 2020; Kim et al, 2009). Importantly, the prelimbic subregion of mPFC remains immature after birth and develops, undergoing a process of programmed synaptic pruning followed by regulation of synaptic and neuronal function which can persist into adulthood and continue organization of maturation circuitry (Burgos-Robles et al, 2009; Sotres-Bayon et al, 2012).

Convergent adolescent rodent contextual fear findings further highlight the importance of the developing prelimbic cortex in mediating fear responses. For instance, during very early development in pre-weanling rodents, odor-shock conditioning can be modulated by maternal presence (Moriceau et al, 2006), and the mechanisms of cued extinction learning differ from adult-like extinction via alterations in N-methyl-D-aspartate (NMDA) receptors (NMDARs) requirement (Langton et al, 2007) and mPFC activity (Kim et al, 2009), suggestive of the perineuronal nets protect fear memories from degradation and erasure (Gogolla et al, 
2009; Kim and Richardson, 2007). Potentiation of the prelimbic excitatory synapses after fear acquisition in postnatal day (PD) 23 mice, and its subsequent depotentiation upon extinction, suggest that the prelimbic region excitatory synapses dynamically regulate fear expression (Pattwell et al, 2012). Furthermore, the deep-layer prelimbic pyramidal neurons of juvenile animals can exert suppressive effects on fear-enhancing potentiation through the selective elimination of principally excitatory synapses (Pattwell et al, 2011; Pattwell et al, 2016). More convincingly, the mPFC shows enhanced functional connectivity during extinction of the reminded fear may play a role in enabling extinction learning training to more persistently modify the original threat-memory trace (Schiller et al, 2013). Therefore, understanding the underlying mechanism in memory destabilization upon reactivation may shed light on treatments for neurodevelopmental disorders, such as schizophrenia-spectrum and primary psychotic disorders, mood disorders and post-traumatic stress disorder (PTSD).

In stark contrast to adult animals, fear extinction of rats younger than 3 weeks appears to be permanent and has been suggested to reflect an unlearning process that leads to the erasure of previously conditioned fear memories (Kim and Richardson, 2008). Recent study has shown that the modification of an existing fear memory trace was associated with the retrieval-dependent alpha-amino-3-hydroxy-5-methyl-4isoxazole propionate-type glutamate receptors (AMPARs) trafficking (Holehonnur et $a l, 2016)$. Fear circuitry during the retrieval of existing memories has been shown to be particularly plastic, which is a protein-synthesis-dependent process (Finnie and Nader, 2012; Lee, 2009; Tronson and Taylor, 2007). However, the neuronal mechanisms underlying the developmental regulation of retrieval-dependent memory are not fully known.

The precursor form of brain-derived neurotrophic factor (BDNF), proBDNF, which signals through its receptor $\mathrm{p} 75^{\mathrm{NTR}}$, has been shown to modulate structural plasticity, spine pruning and cell apoptosis during brain development. Abundantly proBDNF and its receptor are expressed in developing brain regions exhibiting a high degree of plasticity, such as the cerebellum, hippocampus and mPFC (Menshanov et al, 2015; Sun et al, 2021a; Yang et al, 2009c). During cortical development levels of 
proBDNF rise, the activation of proBDNF-p $75^{\mathrm{NTR}}$ signaling can negatively regulate neural remodeling by selectively facilitating NMDAR-dependent neurotransmission (Yang et al, 2014) and neuronal activity (Sun et al, 2019). Recently, we found that infusions of proBDNF in the infralimbic region facilitated induction of fear extinction, while infusions in the prelimbic region depressed fear expression (Sun et al, 2019; Sun et al, 2018a). This proBDNF-induced extinction is sufficient for extinguishing both new and older memories (Sun et al, 2018a). Indeed, prefrontal proBDNF is able to elevate neuronal correlate during the acquisition of conditioned extinction and facilitate dorsolateral striatum-dependent flexible behaviors (Sun et al, 2020b). Intriguingly, circadian proBDNF in the projection of the hippocampo-prefrontal cortex pathway possible engage in regulation of sleep homeostasis to extinguishing original memory (Sun et al, 2019). More recent research demonstrated that the peak expression of proBDNF during the $4^{\text {th }}$ postnatal week promoted the NMDAR-dependent short-lasting long-term depression (LTD), which was accompanied by inhibiting neuronal migration and axonal retraction (Sun et al, 2021a; $\mathrm{Xu}$ et al, 2011; Yang et al, 2009a). These retrograde effects of proBDNF on dendritic complexity and spine density are strongly linked to memory decay (Kasai et al, 2010).

Although previous reports provide important insights into pro-neurotrophic mechanisms for regulating fear extinction and weakening unwanted memory (Gibon et al, 2015; Yang et al, 2014; Yang et al, 2009c), it remains unclear whether and how prelimbic proBDNF regulate retrieval-dependent memory destabilization, especially when its high expression is observed during the early development stage. This study tested the hypothesis that the high expression of prelimbic proBDNF in the juvenile rodents influenced the modification of the weak fear memory traces. Furthermore, the experiments described here investigated the possible role of proBDNF in synaptic development and neuronal function via the $\mathrm{p} 75^{\mathrm{NTR}}$ and NMDAR signaling. These findings may help to understand the characterization of developmentally regulates synaptic and neuronal functions underlying inherently suppressing unwanted fearful memories. 


\section{Materials and Methods}

\subsection{Animals}

All procedures were approved by the Care and Use of Animals Committee of Guizhou University of Traditional Chinese Medicine (SCXK-2013-0020). Juvenile (PD24-PD26) and adult (PD72-78) male Wistar rats (Beijing Vital River Laboratory Animal Technology Co., Ltd., China) were group-housed (two-four rat/cage) and reared under an environmentally controlled room $\left(21 \pm 2^{\circ} \mathrm{C} ; 45 \pm 5 \%\right.$ humidity $)$ and maintained in a 12-h light/dark cycle with food and water available ad libitum. To minimize possible circadian influences on learning and memory processing, all experiments were conducted during the light period (between 1400 and 1700). Handled extensively (10 min per day for at least two days) rats were averagely and randomly received one of infusion or co-infusion to excluded drug accumulation effects.

\subsection{Behavioral manupulations}

Using the same behavioral paradigm as in our previous studies, conditioned freezing behavior was tested in operant chambers (Coulbourn Instruments, Pennsylvania, USA) enclosed in ventilated sound-attenuating boxes (Med Associates Inc, Vermont, USA) with video system, which was supported by a video camera for scoring of animal's behavior. Each chamber was illuminated by a dimly light and auditory stimuli were delivered from a programmable high-frequency speaker (ENV-224BM). Electrical footshock $(2.0 \mathrm{~s}, 0.75 \mathrm{~mA})$ was delivered through a grid floor via a constant current aversive stimulator (ENV-414S). The expression of fear behavior was automatically measured by the Med Associates software.

The procedure for four or eight tone-shock fear conditioning was referred a previously published model (Holehonnur et al, 2016; Wang et al, 2009). Rats were allowed to acclimate to the training apparatus for $150 \mathrm{~s}$, followed by the presentation of a habituation tone $(4 \mathrm{kHz}, 30 \mathrm{~s}, 77 \mathrm{~dB})$ with a footshock. Four tone-shock fear training used an ITI of 2 min and eight tone-shock fear training used a random but averaged 4 min ITI (range from 1 to $7 \mathrm{~min}$ ). Rats were removed from the training chamber $30 \mathrm{~s}$ after the delivery of the last footshock and returned to their home cages. 
Fear memory test was performed one day after conditioning in a novel chamber with distinct environment (including tactile, visual, and olfactory cues). The test consisted of a $150 \mathrm{~s}$ acclimation period, followed by the presentation of four 30-s tone presentations with 2-min ITIs. Percentage freezing was measured during each tone presentation.

Five days after fear conditioning, rats were subjected to fear memory retrieval by exposing to a novel chamber with distinct environment (including tactile, visual, and olfactory cues). They were subjected to a $150 \mathrm{~s}$ acclimation period, were presented with one tone presentation for $30 \mathrm{~s}$, and then were placed back to their home cage $30 \mathrm{~s}$ after the tone presentation. Percentage freezing was measured during the tone presentation. Post-retrieval short-term memory (PR-STM) and post-retrieval long-term memory (PR-STM) occurred 30 min and 2 days after the memory retrieval in a novel chamber with distinct cues, respectively. They were subjected to a $150 \mathrm{~s}$ acclimation period and four 30-s tone presentations with 2-min ITIs, and then were placed back to their home cage $30 \mathrm{~s}$ after the last tone presentation. Percentage freezing was measured during each tone presentation.

For fear extinction, rats were subjected to a $150 \mathrm{~s}$ acclimation period, followed by the presentation of eight 30-s tone presentations with 2-min ITIs five days after the fear conditioning. Percentage freezing was measured during each tone presentation. For the expression of fear, freezing behavior was reported as the freezing behavior during the first three tones. Fear reinstatement was conducted one day following the extinction training. During the reinstatement training, rats were subjected to two non-contingent footshock trials with a 1-min ITI. Percentage freezing was measured during each tone presentation.

\subsection{Stereotaxic surgery and infusion}

Rats were anesthetized with isoflurane and placed in a stereotaxic frame (SN-3, Narishige, Japan) for surgery. Stainless steel guide cannulae (22 gauge; Plastics One, Inc.) were bilaterally inserted above the prelimbic region of the mPFC (AP: $+3.2 \mathrm{~mm}$, ML: $\pm 0.6 \mathrm{~mm}, \mathrm{DV}: 3.2-3.6 \mathrm{~mm}$ ). A sterile stainless steel stylet (30-gauge, $10 \mathrm{~mm}$, Plastics One Inc.) was inserted into guide cannula to avoid obstruction. 
During infusions, rats were gently restrained and infusions were achieved by inserting 30-gauge needles (10 mm, Small Parts Inc.) connected through PE-50 tube into a microsyringe pump (Harvard Apparatus), extended $1.0 \mathrm{~mm}$ beyond the end of the cannulae. Needles were inserted into bilateral cannulae and then cleavage-resistant proBDNF (2 ng/ml; Cat\#B257 Alomone Labs), anti-proBDNF antibody (10 $\mu \mathrm{g} / \mu \mathrm{L}$; Cat\#ANT-006, Alomone Labs), TAT-Pep5 (4 ng/ $\mu \mathrm{L}$; Cat\#506181, EMD Millipore), $\mathrm{K} 252 \mathrm{a}$ $\mu \mathrm{g} / \mu \mathrm{L}$; Cat\#82497;

Sigma-Aldrich), 3-(2-carboxypiperazin-4-yl)propyl-1-phosphonic acid (CPP; $32 \mathrm{ng} / \mu \mathrm{L}$; Cat\#01773, Tocris Bioscience), NVP-AAM077 (0.8 ng/ $\mu \mathrm{L} ;$ Cat\#P1999, Sigma-Aldrich), Ro25-6981 (2.0 ng/ $\mu \mathrm{L}$; Cat\#1594, Tocris Bioscience), mature BDNF (1.5 $\mu \mathrm{g} / \mathrm{mL}$; Cat\#B250; Alomone Labs) or artificial CSF (ACSF, Cat\#3525, Tocris Bioscience) into prelimbic area (at a rate of $0.5 \mu \mathrm{L} / \mathrm{min} /$ side for $2 \mathrm{~min}$ ) was infused immediately or one day following memory retrieval. The injection cannulae were left in place for 3-5 min before being slowly retracted. Dose and route of administration were chosen as described previously (An et al, 2018a; An and Sun, 2018b; Sun et al, 2018a). Rats were weighed and monitored at least three days to ensure recovery. One day before commencing experiment, a habituation session was performed three times for each rat without any infusion.

\subsection{Tissue preparations and Western blot analysis}

According to the previous principle (Sun et al, 2021c; Sun et al, 2021d), prelimbic cortex were dissected and homogenized in lysis buffer ( $\mathrm{pH}$ 7.4) containing a cocktail of protein phosphatase and proteinase inhibitors (Sigma). The samples were centrifuged at 14,000 r.p.m. for $15 \mathrm{~min}$ at $4^{\circ} \mathrm{C}$ and the supernatant was collected. Protein was quantified and normalized to the bicinchoninic acid (BCA) assay. Protein amounts $(20 \mu \mathrm{g})$ were resolved in 10-15 \% SDS-PAGE gels followed by blotting onto polyvinylidene fluoride membranes (Pall, Pensacola). Samples were incubated with the primary antibody: mouse anti-proBDNF (1:500; Cat\#sc-65514, Santa Cruz Biotechnology), rabbit anti-p75 ${ }^{\mathrm{NTR}}(1: 1,000 ;$ Cat\#AB1554, Chemicon), rabbit antiGluN2A (1:1000; Cat\#07632, Millipore), mouse anti-GluN2B antibody (1:1000; Cat\#06600, Millipore), mouse anti- $\beta$-actin (1:20,000; Cat\#A5316, Sigma) overnight 
at $4^{\circ} \mathrm{C}$. After further incubation in horseradish-peroxidase (HRP)-conjugated secondary goat anti-mouse (1:2500; Cat\#31430, Thermo Fisher Scientific) or anti-rabbit (1:2500; Cat\#31460, Thermo Fisher Scientific) IgG for two hours at room temperature, immunoreactivity was detected by ECL Western Blotting Detection Kit (CWBIO, China). The relative values were calculated and normalized to the corresponding $\beta$-actin signal. The expression of the target proteins in juvenile group were presented as fold changes relative to the appropriate control values obtained from adult animals.

\subsection{Spine density analysis}

Rats were anesthetized by an intraperitoneal injection of sodium pentobarbital ( 80 $\mathrm{mg} / \mathrm{kg}$ ). The brains were removed, rinsed in phosphate-buffered saline (PBS), and stained using Golgi-Cox method, according to the manufacturer's instructions (Rapid GolgiStain; FD Neurotechnologies). The brain was blocked and placed immediately into Golgi impregnation solution (a mixture of equal volumes of solution A and B provided in the kit) in the dark. The impregnation solution was replaced once after 24 hours and stored in the dark for two or three weeks at room temperature. Brain tissue was then transferred to a cryoprotectant solution (solution C) for one week at $4{ }^{\circ} \mathrm{C}$. Tissues were frozen, embedded in OCT (ThermoFisher), and sectioned into $100 \mu \mathrm{m}$ thick. Following air-drying in the dark, sections were rinsed with distilled water reacted in a developing solution, and dehydrated in 50\%, 75\%, 95\%, and $100 \%$ ethanol for 5 min each. The slides were mounted under a coverslip with Permount and allowed to dry in the air. The region of interest was viewed and traced using a BX51 Olympus microscope (100xobjective) attached to Olympus DP50 camera and reconstructed using Neurolucida 8.0 software (MBF Bioscience). All clearly evaluable areas of brain slices, which containing 50-150 $\mu \mathrm{m}$ of secondary dendrites from each imaged soma in layer V/VI were selected as previously described (Cerqueira et al, 2007; Moench and Wellman, 2017). Three pyramidal neurons per section and three sections per animal were analyzed. For spine categorization, the following criteria were used (Li et al, 2017; Sun et al, 2020b; Sun et al, 2021a): (1) 
mushroom: spine head diameter was $\geq 1.5 \times$ spine neck diameter; (2) stubby: spine head and spine neck were roughly of same width, and spine length was not significantly longer than head diameter; (3) thin: spine head and spine neck were roughly of same width, and spine length was much longer than spine head width. Spine densities were calculated using NeuroExplorer software (MBF Bioscience) and expressed as the mean number of spines per micrometer dendrite.

\subsection{Patch-clamp recording}

As previously described (An and Sun, 2017; Li et al, 2018; Sun et al, 2021e), animals were decapitated and the brains were removed to an ice-cold, oxygenated (95\% $\mathrm{O}_{2}$ and $5 \% \mathrm{CO}_{2}$ ) high-sucrose solution that contained $200 \mathrm{mM}$ Sucrose, $1.9 \mathrm{mM} \mathrm{KCl}$, $1.2 \mathrm{mM} \mathrm{NaH}_{2} \mathrm{PO}_{4}, 33.0 \mathrm{mM} \mathrm{NaHCO} 3,10.0 \mathrm{mM}$ glucose, $4.0 \mathrm{mM} \mathrm{MgCl}_{2}$ and $0.7 \mathrm{mM}$ $\mathrm{CaCl}_{2}$, pH 7.4 (with an osmolarity of 300-305 mOsm). Horizontal slices (300 $\mu \mathrm{m}$ thickness) were prepared with a vibratome (VT1000S, Leica, Germany). After a one hour recovery period, slices were recorded in a chamber mounted on a contrast-enhanced CCD camera (Hamamatsu) equipped with infrared gradient contrast. The slices were perfused with a continuous flow of ACSF $\left(95 \% \mathrm{O}_{2}\right.$ and $5 \%$ $\mathrm{CO}_{2}$ ) that contained $120 \mathrm{mM} \mathrm{NaCl}, 3.5 \mathrm{mM} \mathrm{KCl}, 1.25 \mathrm{mM} \mathrm{NaH} \mathrm{PO}_{4}, 26.0 \mathrm{mM}$ $\mathrm{NaHCO}_{3}, 10.0 \mathrm{mM}$ glucose, $1.3 \mathrm{mM} \mathrm{MgCl}$ and $2.5 \mathrm{mM} \mathrm{CaCl}_{2}, \mathrm{pH}$ 7.4.

Whole-cell voltage-clamp recordings were performed in pyramidal neurons in layer V/VI of prelimbic region using pipettes with 3-7 M resistance after being filled with pipette solution containing (in $\mathrm{mM}$ ) K-glu 130, $\mathrm{MgCl}_{2}$ 2, HEPES 10, EGTA 3, $\mathrm{Na}_{2}$-ATP 2, PH 7.3. The pipettes were pulled using a P-97 electrode puller (Sutter Instruments). All the cells were held at $-70 \mathrm{mV}$, then slow and fast capacitance compensation was automatically performed. Access resistance was continuously monitored during the experiments. Neurons were considered only when the seal resistance was $>500 \mathrm{M} \Omega$ and the series resistance $(<30 \mathrm{M} \Omega)$ changed $<20 \%$ throughout the experiment. Positive pressure was applied to the recording pipette as it lowered into the medium and approached the cell membrane. Constant negative pressure was applied to form the seal $(>1 G \Omega)$ when the recording pipette attached to 
the membrane. And then suck quickly to rupture the cell membrane and access whole cell configuration. All experiments were carried out at room temperature $\left(22 \pm 1^{\circ} \mathrm{C}\right)$. Data of EPSC were recorded using an EPC-10 patch-clamp amplifier (HEKA Instruments). Signals were digitized at $10 \mathrm{kHz}$, low-pass filtered at $2.9 \mathrm{kHz}$, stored on a disk using Pulse software (HEKA, Germany). Synaptic spontaneous excitatory postsynaptic currents (sEPSCs) were isolated by bath application bicuculline $(10 \mu \mathrm{M})$ to block the gamma-aminobutyric acid type $\mathrm{A}\left(\mathrm{GABA}_{\mathrm{A}}\right)$ receptor mediated synaptic currents. Only one slice from each rat was used for final analyses. Spike curves were analyzed using Clampfit software (Molecular Devices). Decay time was calculated in Axograph $\mathrm{X}$ using a two-exponential simplex fit.

\subsection{In vivo long-term depression}

Thirty min after the memory retrieval or infusions, field excitatory postsynaptic potentials (fEPSPs) in at the hippocampal-prefrontal cortex pathway were recorded as published methods (Sun et al, 2020a; Takita et al, 2013; Thomases et al, 2014). Following craniotomies above the region of the $\mathrm{mPFC}$ and hippocampus, monopolar electrodes (insulated platinum iridium wire, AM Systems) and tungsten bipolar stimulating electrodes (FHC) were implanted into prelimbic region (AP: $+3.2 \mathrm{~mm}$, ML: $\pm 0.6 \mathrm{~mm}, \mathrm{DV}: 3.2-3.6 \mathrm{~mm}$ ) and ventral hippocampus (AP: $-6.0 \mathrm{~mm}, \mathrm{ML}: \pm 5.2$ $\mathrm{mm}, \mathrm{DV}:$ 6.5-7.0 $\mathrm{mm}$ ) regions, respectively. The cerebral hemisphere was chosen pseudorandomly for each animal. Neural signal was amplified $(\times 100)$, filtered at 5-5000 Hz, digitized and collected at $20 \mathrm{kHz}$ sample frequency (Scope software, PowerLab; AD Instruments). After optimal placement of the stimulating (100 ms pulse width, squared monophasic pulse) and recording electrodes, stimulus intensity was adjusted to about $50 \%$ of maximal fEPSP slope. Baseline fEPSPs were recorded every $60 \mathrm{~s}$ for at least $20 \mathrm{~min}$. Long-term depression (LTD) was induced by a low-frequency stimulation (LFS; 300 pulses at $1.0 \mathrm{~Hz}$ ). Initial data measurement was performed in Clampfit 9.0 (Molecular Devices). The fEPSPs slope was used to measure synaptic efficacy. The fEPSP slope at every time point was normalized to the mean fEPSP slope during the baseline period. Comparisons among the last $20 \mathrm{~min}$ of the 60-min decay were used to analyze. 


\subsection{LFP recording}

Four or five days before behavioral training, microelectrode implantation was conducted using previously reported procedures (Sun et al, 2019; Sun et al, 2018b; Sun et al, 2021b). Rats were anesthetized with isoflurane during the surgery. An impedance-measured $(200-600 \mathrm{k} \Omega)$ microelectrode, which was arrayed into a $4 \times 8$ matrix using 25 - $\mu$ m-diameter tungsten wires (California Fine Wires) in a 35-gauge silica tube (World Precision Instruments). A cannula was attached to a silica tube. The proximal open end of the cannula was parallel to electrode tips. The electrode array was chronically inserted into prelimbic region (AP: $+3.2 \mathrm{~mm}, \mathrm{ML}: \pm 0.6 \mathrm{~mm}, \mathrm{DV}$ : 3.2-3.6 mm). Left or right hemisphere was implanted randomly but counterbalanced designed. The headmount was fastened to the cranium by dental acrylic with scull screws. A stainless steel wire was affixed to the skull to act as reference and ground electrodes.

Data were acquired on a Digital Cheetah system (Cheetah software, Neuralynx Inc.). Unit signals were recorded via a HS-36 unit gain headstage (Neuralynx Inc.) mounted on animal's head by means of lightweight cabling that passed through a commutator (Neuralynx Inc.). LFPs were sampled at $32 \mathrm{kHz}$ and filtered at $0.1-9,000$ $\mathrm{Hz}$ from each electrode. Neural signals were transferred through a slip-ring commutator (Neuralynx) to the data acquisition system.

The LFPs during the memory retrieval were collected for further analysis, which was performed by using Neuroexplorer (Nex Technologies). The power of each frequency was calculated using Welch's method (1024 frequencies between 1 and 200 $\mathrm{Hz}$, smoothed with a Gaussian Kernel with bin width 3). The band power of theta and gamma were defined as the mean power in the frequency range of 6-12 Hz and 50-80 $\mathrm{Hz}$, respectively.

To quantify the amplitude modulation by phase, Tort's modulation index (MI) was calculated using a normalized entropy measure previously described (Sun et al, 2018b; Tort et al, 2008; Voloh et al, 2015). Briefly, the preprocessed voltage trace from a single trial, $x_{\text {raw }}(t)$ (raw signal), was filtered into two frequency bands of interest $f_{P}$ 
and $f_{A}$, generating $x_{f P}(t)$ and $x_{f A}(t)$, respectively. The standard Hilbert transform was applied to extract the time series of the phase $\varphi_{f P}(t)$ and amplitude envelope $A_{f A}(t)$ from $x_{f P}(t)$ and $x_{f A}(t)$, respectively. The composite time series $\left[\varphi_{f P}(t), A_{f A}(t)\right]$ were then constructed, which calculated the amplitude of the $f_{A}$ phase onto each phase of the $f_{P}$ rhythm. The phase $\varphi_{f P}(t)$ were binned into interval of phase $(n=18 ; 20$ intervals). The mean amplitude $\left(A_{f A}\right)_{\varphi_{f p}}(j)$ of $A_{f A}(j)$ was computed over each phase bin $j$. The entropy measure $H$ was calculated as follows:

$$
H=-\sum_{j=1}^{N} \frac{\left(A_{f A}\right) \varphi_{f p}(j)}{\sum_{j=1}^{N}\left(A_{f A}\right)_{f p}(j)} \cdot \log \frac{\left(A_{f A}\right)_{f p}(j)}{\sum_{j=1}^{N}\left(A_{f A}\right)_{f p}(j)}
$$

Where $N=18$, which was equal to the number of bins.

The null hypothesis of the test is that the expected amplitude distribution is uniform.

Thus, the $\mathrm{MI}$ is the normalized $\mathrm{H}$ by the uniform distribution $(\log (N))$,

$$
M I=\frac{\log (N)-H}{\log (N)}
$$

The range of MI value was larger than zero. A value above zero indicated the phase-to-amplitude modulation. The stronger phase-to-amplitude modulation, the larger MI value would be.

\subsection{Statistical analysis}

Data are expressed as mean \pm S.E.M.. All analyses were performed with Neuroexplorer and SPSS 17.0 software. One-Sample Student t-tests were used to compare data from western-blot, spine density and fear expression. Other data were analyzed with one-way or two-way repeated analysis of variance (ANOVA). Post hoc analyses were performed with Tukey's test where appropriate. $P<0.05$ level of confidence were used in the analyses. The number of animals in each group and other details can be found in figure legends and results. 


\section{Results}

\subsection{The increase proBDNF during the juvenile period enhances} retrieval-dependent fear memory destabilization.

The expression of proBDNF and its receptor $\mathrm{p} 75^{\mathrm{NTR}}$ in the prelimbic region of mPFC were detected in un-manipulated animals. Both proBDNF (Figure 1A; T-test, $t_{10}=2.54, P=0.030$ ) and $\mathrm{p} 75^{\mathrm{NTR}}$ (Figure $1 \mathrm{~B}$; T-test, $t_{10}=2.32, P=0.043$ ) levels of juvenile rats are significantly higher than adults. To investigate whether different intensity of auditory fear conditioning affect memory strength, rats were fear conditioned with either four tones or eight tones. The eight-tone groups of both juvenile and adult rats exhibited significantly higher tone-induced freezing than the four-tone groups (Figure 1C; two-way ANOVA, effect of tone: $F_{(1,18)}=5.39, P=0.032$ ) without age differences (effect of age: $F_{(3,18)}=0.13, P=0.941$ ), indicating that the eight-tone training resulted in stronger memories. To determine whether the increase proBDNF during the juvenile period was involved in fear memory destabilization, rats were bilaterally infused with proBDNF (Adult+Pro, Juvenile+Pro), anti-proBDNF antibody (Adult+Anti, Juvenile+Anti), anisomycin (Juvenile+Anis) or vehicle (Adult, Juvenile) immediately following memory retrieval. For rats trained with four tones, repeated-measures ANOVA indicated no statistical difference in freezing levels among groups during memory retrieval (Figure 1D). However, there was a significant decrease in freezing in the juvenile group compared with adult group during PR-LTM test (repeated-measures ANOVA, interaction effect of between treatment and test: $F_{(5}$, $31)=4.21, P=0.005$; post-hoc, Juvenile or Adult, $P<0.05$ ), which was conducted two days after memory retrieval. For the juvenile groups, infusion proBDNF or anisomycin depressed freezing behavior (post-hoc, Juvenile vs. Juvenile+Anis or Juvenile+Pro, both $P<0.05$ ), while infusion anti-proBDNF antibody enhanced the freezing levels (post-hoc, Juvenile or Juvenile+Anti, $P<0.05$ ). However, proBDNF-infused adult rats did not display less freezing (post-hoc, Adult vs. Adult+Pro, $P>0.05)$. Therefore, endogenous proBDNF of juvenile rats facilitated retrieval-dependent destabilization, while exogenous proBDNF could regulate the memory destabilization of juvenile but not adult animals. For rats trained with eight 
tones, repeated-measures ANOVA revealed no statistical difference in freezing levels among groups during memory retrieval or PR-LTM test (Figure 1E; repeated-measures ANOVA, interaction effect of between treatment and test: $F_{(5,}$ ${ }_{24}=0.22, P=0.951$ ). Consistent with previous reports (Holehonnur et al, 2016; Wang et $a l, 2009)$, these findings imply that strong memories formed via eight-tone training do not undergo retrieval-dependent memory destabilization. To investigate whether the memory destabilization was resulted from changes in PR-STM, post-retrieval tests were conducted in rats trained with four tones 30 min following the memory retrieval. There was no interaction effect of between treatment and test on the freezing level (Figure 1F; repeated-measures ANOVA, interaction effect of between treatment and test: $\left.F_{(5,20)}=0.18, P=0.967\right)$. Meanwhile, proBDNF, its anti-body or anisomycin effects on the freezing behavior during the PR-LTM tests were not observed when juvenile rats trained four tones were infused one day after memory retrieval (Figure $1 \mathrm{G}$; repeated-measures ANOVA, interaction effect of between treatment and test: $F_{(5}$, ${ }_{20)}=2.93, P=0.038$; post-hoc, Juvenile vs. Juvenile+Pro or Juvenlie+Anti or Juvenile+Anis, all $P>0.05$ ), suggesting there was no remote effect of proBDNF or anisomycin on post-retrieval memory during the juvenile period. Similarly, no significant difference was found between Adult and Adult+Pro group during memory retrieval (post-hoc, Adult vs. Adult+Pro, $P>0.05$ ) or PR-LTM test (post-hoc, Adult vs. Adult+Pro, $P>0.05)$. Together, these results indicate the high expression of endogenic proBDNF in the juvenile rat prelimbic cortex plays a central role in promoting retrieval-dependent memory destabilization of a weak fear memory trace.

Rats trained with four tones were subjected to fear extinction training five days after fear conditioning. There was no significant main effect of age on acquisition of fear memory extinction (Figure $1 \mathrm{H}$; repeated-measures ANOVA, effect of age: $F_{(1}$, $\left.{ }_{8}=0.017, P=0.898\right)$. The inhibited freezing levels of both juvenile and adult groups could be completely regained when a reinstatement training was conducted one day following fear extinction (repeated-measures ANOVA, effect of age: $F_{(1,8)}=0.021$, $P=0.889$ ). Together, these findings imply that the high level of proBDNF expression during the juvenile period did not inhibit fear extinction or expression, or degrade the 
original fear memory. Meanwhile, there was no statistical difference in the mean fear expression of the first three tones during extinction training (Figure 1I; T-test, $t_{8}=0.19$, $P=0.858)$. We also ruled out the possible difference in locomotion, anxiety or motivation between juvenile and adult as rats had a similar travel distance (Supplementary Figure S1B) and the proportion time in the center field (Supplementary Figure S1C) during the open field test, and a similar motivation behavior in the lever-press task (Supplementary Figure S1D). Therefore, the performance of juvenile rats during the PR-LTM test is not attributed to the alteration of locomotion, anxiety or motivation.

\subsection{The proBDNF/p75 $75^{\mathrm{NTR}}$ signaling mediated by the activation of GluN2B is involved in retrieval-dependent memory destabilization.}

To further confirm if the proBDNF-mediated memory destabilization involved in $\mathrm{p} 75^{\mathrm{NTR}}$ or $\operatorname{TrkB}$ pathway, $\mathrm{p} 75^{\mathrm{NTR}}$ (TAT-Pep5) or TrkB (K252a) inhibitor was bilaterally infused immediately following memory retrieval. Blocking prelimbic p $75^{\text {NTR }}$ by the TAT-Pep5 inhibitor significantly enhanced the freezing level during the PR-LTM test (Figure 2A; repeated-measures ANOVA, interaction effect of between group and day: $F_{(5,23)}=3.34, \quad P=0.021$; post-hoc, Juvenile(Con+Con) vs. Juvenile(Pep5+Con), $P<0.05)$. Meanwhile, the effects of exogenous proBDNF could be prevented by administration of TAT-Pep5 (post-hoc, Juvenile(Con+Pro) vs. Juvenile(Pep5+Pro), $P<0.05$ ), but not K252a (post-hoc, Juvenile(Con+Pro) vs. Juvenile(K252a+Pro), $\quad P<0.05)$, indicating that retrieval-dependent memory destabilization was associated with the proBDNF/p $75^{\mathrm{NTR}}$-mediated pathway. To detect whether neutralizing proBDNF with its antibody would potentially interfere with the expression of endogenous protein and its proteolysis to mBDNF, rats were infused with mBDNF following memory retrieval. The freezing level of mBDNF-infused rats was similar to vehicle group during the PR-LTM test (post-hoc, Juvenile(Con+Con) vs. Juvenile(Con+mBDNF), $P>0.05)$. These observations following the infusion reflected merely the proBDNF effect rather than the mBDNF effect. The findings clearly indicate that proBDNF-p $75^{\mathrm{NTR}}$ signaling influences the modification of an existing memory trace. 
Growing evidences suggest that NMDAR activity at the very moment of memory retrieval play an important role in updating reconsolidation and inducing memory destabilization (Ben Mamou et al, 2006; Holehonnur et al, 2016; Milton et al, 2008). Meanwhile, previous studies indicated that BDNF-induced fear extinction and memory consolidation were linked with the activation of NMDA receptors (Ma et al, 2021; Sotres-Bayon et al, 2009; Sun et al, 2019; Sun et al, 2018a). Furthermore, depending on the age of the animals, the dynamic changes in the expression of GluN1, GluN2A, and GluN2B subunit mRNAs can lead to different mixtures of NMDA receptors during development of rat cortex (Liu et al, 2004; Ohno et al, 2010; Sheng et al, 1994). To determine whether the proBDNF-mediated memory destabilization was regulated by NMDA receptors, we first examined the levels of NMDA-GluN2A and NMDA-GluN2B receptors in prelimbic cortex of both adult and juvenile rats. Then juvenile rats were subjected to four-tone fear conditioning as above, followed by infusion of NMDA (CPP), NMDA-GluN2A (NVP-AAM077) or NMDA-GluN2B (Ro25-6981) receptor antagonist into the prelimbic region immediately after memory retrieval. The NMDA-GluN2A level of juvenile group was significant lower than adult group (Figure 2B; T-test, $t_{10}=2.86, P=0.017$ ) while a higher level of NMDA-GluN2B in the juvenile group was found (Figure 2C; T-test, $t_{10}=2.69$, $P=0.019$ ). Both CPP and Ro25 enhanced the fear behavior during PR-LTM test (Figure 2D; repeated-measures ANOVA, interaction effect of between group and test: $F_{(5,29)}=2.87, P=0.032$; post-hoc, Juvenile(Con+Con) vs. Juvenile(CPP+Con) or Juvenile $($ Ro25+Con), both $P<0.05)$ but NVP-treated rats exhibited a similar freezing level to vehicle rats (post-hoc, Juvenile(Con+Con) vs. Juvenile(NVP+Con), $P>0.05)$. Furthermore, the proBDNF effects on memory destabilization could be effectively blocked by GluN2B antagonist Ro25 (post-hoc, Juvenile(Con+Con) vs. Juvenile(Ro25+proBDNF), $P<0.05)$. These results demonstrate the involvement of proBDNF-NR2B pathway in proBDNF-mediated the induction of reconsolidation updating.

3.3 The changes in synaptic and neuronal function promote the modification of the existing fear memory trace. 
Previous evidence supports that proBDNF signaling plays a critical role in synapse formation during early brain development (Je et al, 2012; Langlois et al, 2013; Orefice et al, 2016; Sun et al, 2021a). Furthermore, dendritic spine constitutes the main locus of excitatory synaptic interaction among central neurons. The great variety of shapes and abundance has focused considerable attention on the dendritic spine as the key site for the potential encoding of activity-dependent, neuronal plasticity (Engert and Bonhoeffer, 1999; Weber et al, 2016). Given the relation between proBDNF and synaptogenesis during early brain development, it is important to evaluate the proportion of dendritic spine, neuronal and synaptic function during the early postnatal period, which may help understand the mechanism of retrieval-dependent memory destabilization.

Six hours after the memory retrieval, prelimbic cortex was collected and analyzed alterations in dendritic morphology. For the total density of dendritic spines, no significant difference was observed between juvenile and juvenile+anti rats (Figure $3 \mathrm{~A}$ (bottom-left); T-test, $t_{10}=0.27, P=0.403$ ). However, the percentage of mushroom spine types was significant lower in the juvenile group in comparison to the anti-proBDNF group (Figure $3 \mathrm{~A}$ (bottom-right); T-test, $t_{10}=2.29, P=0.047$ ) while the proportion of thin spines of juvenile rats was significant higher than juvenile+anti group (Figure 3A(bottom-right); T-test, $t_{10}=2.33, P=0.042$ ). No statistical difference was found in stubby spines between two groups (Figure 3A(bottom-right); T-test, $\left.t_{10}=0.13, P=0.901\right)$. There was no difference in the mean frequency of NMDA currents (Figure 3B-(bottom-left); one-way ANOVA, $F_{(2,15)}=1.26, P=0.312$ ). However, juvenile rats exhibited significant higher NMDA-EPSC amplitude than adults (Figure 3B-(bottom-middle); one-way ANOVA, $F_{(2,15)}=5.72, P=0.014$; post-hoc, Juvenile vs . Adult, $P<0.05)$ while proBDNF-inactivating antibody could effectively depressed the amplitude of juvenile group (post-hoc, Juvenile vs. Juvenile+Anti, $P<0.05$ ). The decay time of NMDA receptor currents was significantly enhanced in juvenile rats compared with adult rats (Figure 3B-(bottom-right); one-way ANOVA, $F_{(2,15)}=4.95$, $P=0.022$; post-hoc, Juvenile vs. Adult, $P<0.05)$. Blockage of proBDNF significantly shortened the decay time of juvenile group (post-hoc, Juvenile vs. Juvenile+Anti, 
$P<0.05$ ), observing no difference between Adult and Juvenile+Anti groups (post-hoc, Adult vs. Juvenile+Anti, $P>0.05)$.

Figure 3C (bottom-left) showed the NMDA receptor-dependent in vivo LTD at hippocampal-prefrontal cortex synapses. Interestingly, the LFS did successfully induced LTD in juvenile but not adult rats. Herein, at the last 20 min of the recording, the average slope of juvenile group was significantly higher than adult and Juvenile+Anti groups (Figure 3C-(bottom-right); one-way ANOVA, $F_{(4,22)}=3.13$, $P=0.035$; post-hoc, Juvenile vs. Adult or Juvenile+Anti, both $P<0.05$ ). The LFS-induced LTD was completely suppressed by GluN2B antagonist Ro25 but not GluN2A antagonist NVP (post-hoc, Juvenile vs. Juvenile+Ro25, $P<0.05$; Juvenile vs. Juvenile+NVP, $P>0.05$ ), confirming a GluN2B-dependent synaptic plasticity. Additionally, input/output curves were established but the fEPSP slopes of juvenile rats did not differ significantly from adults (Supplementary Figure S2B). Meanwhile, LTD was also induced by a stronger LFS (900 pulses of $1.0 \mathrm{~Hz}$ ) as previous methods (An et al, 2019; An et al, 2017, 2018b). The stronger LFS could induce robust LTD, which were comparable between juvenile and adult groups (Supplementary Figure S2C). Inactivation of proBDNF by its antibody could also effectively block the expression of the LTD in juvenile rats. These findings exclude the basal synaptic transmission effects on the LFS-induced LTD and further confirm a weaker LFS (300 pulses of $1.0 \mathrm{~Hz}$ ) can induce LTD in juvenile but not adult rats. To assess whether and how ventral hippocampus is involved in updating fear memory and synaptic function, four-tone conditioned rats were infused with anti-porBDNF into ventral hippocampus immediately following memory retrieval, and PR-LTM and the LFS-induced LTD in the hippocampal-prefrontal cortex pathway was detected as above (Supplementary Figure S3A). We found the inhibition of proBDNF in the hippocampus did not alter retrieval-dependent memory destabilization (Supplementary Figure S3B). Anti-proBDNF antibody could dramatically depress 300-pulse LFS-induced LTD (Supplementary Figure S3C) but not 900-pulse LFS-induced LTD (Supplementary Figure S3D) in juvenile animals. These results clearly indicate that proBDNF in the ventral hippocampus did not play a critical role in retrieval-dependent fear memory 
destabilization or the expression of LFS-induced LTD.

Figure 4D (top) showed that the gamma oscillation of mPFC neurons tended to phase-lock to the peak of theta oscillation. Gamma amplitudes were coupled to theta oscillation during the PR-LTM test and that this modulation was stronger in juvenile group than in adult group (Figure D-(bottom-right); one-way ANOVA, $F_{(2,14)}=7.53$, $P=0.006$; post-hoc, Juvenile vs. Adult, $P<0.05)$. Blockage of the increase proBDNF did reduce the phase-amplitude coupled theta and gamma oscillations compared to juvenile group (post-hoc, Juvenile vs. Juvenile+Anti, $P<0.05$ ). Furthermore, we found equalized theta (Figure 4D-(bottom-middle); one-way ANOVA, $F_{(2,14)}=0.42, P=0.665$ ) and gamma power (Figure 4D-(bottom-right); one-way ANOVA, $F_{(2,14)}=1.13$, $P=0.351)$ during the PR-LTM test among groups, indicating that the difference in coupling strength reflected an active memory process but was not simply the result of improved phase identification due to the increment in power. 


\section{Discussion}

Growing findings in younger rodents indicate that fear expression may vary across neurodevelopment associated with dynamic alterations in fear-related neural circuits. Memory modification using reconsolidation updating is being examined as one of the potential treatment approaches for attenuating memories associated with neurodevelopmental disorders (Beckers and Kindt, 2017; Liberman et al, 2006; Merikangas et al, 2010). However, the underlying mechanism is still unclear. The involvement of proBDNF in fear memory extinction and expression has been demonstrated. Our previous studies reported that proBDNF was highly expressed during the postnatal period and blocking proBDNF-mediated signaling induced spine development deficits in adult rodents. In this study, we further confirmed the expression levels of proBDNF and $\mathrm{p} 75^{\mathrm{NTR}}$ around the $4^{\text {th }}$ postnatal week were marked higher than the adulthood. Auditory fear memories conditioned with eight tones could induce stronger freeze behaviors than four-tone conditioning. This high expression level of prelimbic proBDNF enhanced the retrieval-induced destabilization of the four-tone conditioned weaker memory traces through activating $\mathrm{p} 75^{\mathrm{NTR}}$ and NMDA-GluN2B signaling. Exogenous proBDNF failed to promote the retrieval-dependent memory destabilization in adults but juveniles, indicating an aged-related specific effect of BDNF (Mattson et al, 2004; von Bohlen und Halbach, 2010). Additionally, infusions of proBDNF or its antibody into the juvenile prelimbic region did not change PR-STM or produce remote effects on PR-LTM. Dendritic morphology of prelimbic neurons found higher proportion of thin type spine than anti-proBDNF condition following memory retrieval. Meanwhile, high GluN2B but low GluN2A levels were observed in juvenile animals. The facilitation of neuronal function was verified as evidences by elevating the amplitude and decay time of the NMDA-mediated currents. The GluN2B- but not GluN2A- dependent LTD at hippocampal-prefrontal cortex pathway was enhanced following the memory retrieval. We also found prelimbic theta-gamma coupling of juvenile rats was significantly strengthened during the PR-LTM test. Inactivation of proBDNF could drastically inhibit retrieval-dependent memory destabilization, suppress synaptic 
depression and reduce the neural correlate to the similar level as adults, providing compelling evidence for the pivotal role of proBDNF in memory retrieval and the mechanism.

The expression proBDNF and $\mathrm{p} 75^{\mathrm{NTR}}$ in rodents is developmentally regulated with high levels in the early developmental period of life, correlating well with the timings of synapse formation and elimination (Bartkowska et al, 2010; Yang et al, 2009c). The negative retrograde effects of proBDNF on neuronal connectivity and cell survival have been strongly linked to the improvement in memory extinction and flexible behavior (Sun et al, 2020b; Sun et al, 2018a; Xu et al, 2011; Yang et al, 2009a). Remarkably, spine dynamics are associated with the spine tip where the spine is in contact with the presynaptic bouton (Bhatt et al, 2009; Kasai et al, 2021). In this study, we found blocking proBDNF expression during the juvenile period selectively elevated thin type spines but reduced mushroom ones following memory retrieval. It has been suggested that mushroom spines represent more stable memory spines, which are expected to maintain the fundamental feature of mature neural circuits (Bourne and Harris, 2007; Yang et al, 2009b). The thin spines formed early during development are highly motile and plastic and have been hypothesized to comprise a specialized subpopulation, distinguished by their contribute to synaptic reorganization and new learning (Bourne et al, 2007; Kasai et al, 2010). Our findings are consistent with previous findings that the rapid decline in extinction learning of young animals coincide with the dynamic reorganization of synaptic spine circuitry within the prelimbic region, but not in infralimbic or frontal association cortex (Pattwell et al, 2016). Crucially, the secreted proBDNF can promote spine maturation by sustained elevation of intracellular calcium influx (Mizoguchi et al, 2021; Orefice et al, 2016), which is important in supporting memory destabilization (Da Silva et al, 2013; Jarome et al, 2016).

Electroconvulsive shock or systemic drug administration given after memory reactivation induces the destabilization for the original memory (Milton et al, 2008; Nader et al, 2000; Wang et al, 2009). Reconsolidation involving protein synthesis is required for retrieved memories to persist. Infusion of the protein synthesis inhibitor 
anisomycin into the amygdala can return fear consolidated memories to a labile state (Nader et al, 2000). We found intra-prelimbic infusions of anisomycin and proBDNF shortly after training prevented consolidation of fear memories. These observations are generally agree with recent findings that blocking of the preforntal ERK/CREB-signaling pathway, which is able to be activated by both endogenous and exogenous BDNF, regulates the transition of memory phase from reconsolidation to extinction and this process functions as a switch that cancels reconsolidation of fear memory (Fukushima et al, 2021). However, this suppression was negligible in the stronger conditioned groups. It is possibly due to the fact that strong memories can be resistant to this approach. It has been believed that the strong training down-regulates GluN2B expression, thereby making the memory insensitive to post-reactivation anisomycin infusions but capable of being expressed normally (Ben Mamou et al, 2006; Wang et al, 2009). In fact, an increase in intensity of auditory fear conditioning training lead to an increase in the GluN2A/GluN2B ratio and that this increase is sufficient to prevent retrieval-dependent memory destabilization and therefore inhibits the induction of reconsolidation (Holehonnur et al, 2016). Further researches identified the distinct roles of NMDARs, indicating GluN2B-NMDARs being required for destabilization and GluN2A-NMDARs being required for restabilization (Milton et al, 2013). An increase in NMDA-GluN2B expression can shift the modification threshold to the left thereby leading a lower threshold for LTD induction (Paoletti et al, 2013). In consistent with this switch in NMDAR subunit composition during the developmental epoch (Liu et al, 2004), the higher GluN2B expression was found during the $3^{\text {rd }}$ postnatal week in this study. Previously, a strong NMDAR-mediated EPSCs was induced by the current threshold LFS protocol, leading calcium influx through NMDAR with GluN2B subunits was sufficient for reaching the threshold for LTD induction in infant (PD11-PD14) rats (Yasuda and Mukai, 2015) and in hippocampal slices infused with proBDNF (Woo et al, 2005). This functional recruitment during early development stage is profoundly influenced by the permissive role of proBDNF on shaping synaptic network, with the best example being the apparent increase in amplitude and decay time of EPSCs (Gibon et 
al, 2015; Mizoguchi et al, 2021; Yang et al, 2009a), making them more susceptible for the occurrence of plastic changes. In prevailing models, the level and kinetics of NMDAR activation determine postsynaptic AMPAR trafficking, which is the critical step lead to memory destabilization (Holehonnur et al, 2016; Hong et al, 2013). In addition, there is a consensus concerning the balance between neuronal excitation and inhibition regulating by proBDNF in basal excitability threshold at developing synapses (Deinhardt and Chao, 2014; Langlois et al, 2013; Riffault et al, 2018), implying that metaplasticity mechanisms may serve to alter the specific reactivation cues necessary to destabilize fear memory (Finnie et al, 2012; Zhang et al, 2018). This provides an interesting avenue for future studies.

BDNF-mediated synchronizes neural activity in the mPFC appears to be critical for extinction memory formation and modulation (Hallock et al, 2019; Hill et al, 2016; Rosas-Vidal et al, 2014). Emerging evidence indicates that gamma oscillatory activity is shown to be heterogeneous during extinction learning, with variations in the strength of gamma power (Courtin et al, 2014; Fenton et al, 2016; Mueller et al, 2014). Actually, the gamma amplitude is modulated by the phase of theta, and it is theorized that theta-gamma phase-amplitude coupling coordinates neural activity at the timescale required for memory processing (Benchenane et al, 2011; Nyhus and Curran, 2010). Previously, the strength gamma-theta coupling increased when animals evaluated choice-relevant information (Lundqvist et al, 2011; Roux and Uhlhaas, 2014), which was essential for the destabilization of fear memory at the moment of recall (Radiske et al, 2020). Impaired fear extinction in Bdnf-e4 mice is accompanied by decreased hippocampal-prefrontal theta phase synchrony during early extinction, as well as increased mPFC activation during extinction recall (Hill et al, 2016), implying that activity-dependent BDNF signaling is critical for regulating oscillatory activity, which may contribute to altered behavior. These behavioral associations may be facilitated in part by proBDNF-mediated oscillatory recruitment of functional neuronal ensembles, such as the firing of regular-spiking neurons during theta oscillations (Sun et al, 2018b). Furthermore, recruitment of neuronal ensembles to specific oscillations is thought to occur in part through subthreshold resonance 
properties of neurons (Stark et al, 2013). This hypothesis is in agreement with findings showing that endogenous proBDNF regulates neurons to amplify their response to the oscillatory phase coupling (An et al, 2018a; Sun et al, 2019). Additionally, the role of proBDNF in neural coherence is in keeping with the known roles in synaptic structure and subthreshold LFS induced LTD. Interestingly, enhancement in proBDNF-mediated spike-field coupling reflects the stabilization of striatum-dependent reversal learning, leading to facilitation of repetitive choices by bridging between previous and current choices (Sun et al, 2020b). Furthermore, functional connectivity within the neural circuitry mediating learned fear inhibition, which includes reciprocal projections between $\mathrm{mPFC}$ and amygdala, involves functionally communicate via synchronized neural oscillations (Chen et al, 2021; Likhtik and Paz, 2015). Intriguingly, $8 \mathrm{~Hz}$ stimulation of amygdala can induce amygdalo-cortical network states only following extinction learning (Ozawa et al, 2020), implying that post-extinction fear memory retrieval is supported by both local and interregional experience-dependent resonance.

In this study, we tested the hypothesis that proBDNF plays a vital role in synaptic function and memory destabilization and therefore, the activation of proBDNF signaling can be used to enhance erasure of reconsolidation-resistant fear memories. However, these actions from the temporal elevated proBDNF are probably limited to an early postnatal time window. Our finding showed that intra-prelimbic infusions of exogenous proBNDF failed to promote the destabilization of an existing fear memory, which was coincided with early findings in the different regulations of psychostimulant in BDNF signaling between juvenile and adolescent rats (Banerjee et al, 2009; Kozisek et al, 2008). Therefore, determining the role of proBDNF in mediating adult neural circuitry underpinning learned fear inhibition is another key issue to investigate. Local $\gamma$-aminobutyric acid (GABA) interneurons in PFC, which regulate learned fear and its extinction, also modulate theta and gamma oscillations locally (Chen et al, 2020; Piantadosi and Floresco, 2014). Importantly, the NMDA-dependent switch of proBDNF/p $75^{\mathrm{NTR}}$ signaling can orchestrate the excitatory/inhibitory developmental sequence leading to depolarizing and excitatory 
actions of GABA in adulthood (Langlois et al, 2013; Riffault et al, 2018). The mechanisms underlying this effect needed to be further determined.

Our findings strongly suggest that the mPFC is engaged in destabilization of consolidated memory during memory retrieval, and the high expression of proBDNF during the juvenile period facilitate this processing via activation of the $\mathrm{p} 75^{\mathrm{NTR}}$ and NMDA-GluN2B signaling. Furthermore, proBDNF-mediated synaptic and neural function is involved in regulation of retrieval-dependent memory. Our results also suggest that phase-amplitude coupling analyses from EEG signals recorded can be useful to verify the actual occurrence of retrieval process and predict the treatment's efficacy. Therefore, elucidating the role of the proBDNF in fear memory modification improves our understanding of the molecular mechanisms that can regulate the induction of destabilization/reconsolidation of the stored memories, as suggested by the trace dominance theory (Eisenberg et al, 2003). 


\section{Acknowledgements}

This work was supported by Grants from the National Natural Science Foundation of China $(32160196 ; 31700929)$ to LA.

\section{Ethics Statement}

All animal experiments and procedures were reviewed and approved by the Experimental Animal Care Committee of Guizhou University of Traditional Chinese Medicine (SCXK-2013-0020).

\section{Data Availability Statements}

The data that support the findings of this study will be available in Supplementary Files and other data are available from the corresponding author upon reasonable request.

\section{Conflict of interest}

There is not a conflict of interest for authors.

\section{Author Contributions}

Conceived and designed the experiments: WS, YY, XC, XLL and LA; performed the experiments: WS, YZM, XLL and XC; analyzed the data: WS, XLL and XC; wrote the manuscript: WS, YY, YZM and LA. 


\section{References}

An L, Li J, Luo L, Huang P, Liu P, Tang C, et al (2019). Prenatal melamine exposure impairs cognitive flexibility and hippocampal synaptic plasticity in adolescent and adult female rats. Pharmacol Biochem Behav 186: 172791.

An L, Li X, Tang C, Xu N, Sun W (2018a). Hippocampal proBDNF facilitates place learning strategy associated with neural activity in rats. Brain Struct Funct 223(9): 4099-4113.

An L, Sun W (2017). Prenatal melamine exposure impairs spatial cognition and hippocampal synaptic plasticity by presynaptic and postsynaptic inhibition of glutamatergic transmission in adolescent offspring. Toxicology Letters 269: 55-64.

An L, Sun W (2018b). Acute Melamine Affects Spatial Memory Consolidation via Inhibiting Hippocampal NMDAR-Dependent LTD in Rats. Toxicol Sci 163(2): 385-396.

Banerjee PS, Aston J, Khundakar AA, Zetterstrom TS (2009). Differential regulation of psychostimulant-induced gene expression of brain derived neurotrophic factor and the immediate-early gene Arc in the juvenile and adult brain. Eur J Neurosci 29(3): 465-476.

Bartkowska K, Turlejski K, Djavadian RL (2010). Neurotrophins and their receptors in early development of the mammalian nervous system. Acta Neurobiol Exp (Wars) 70(4): 454-467.

Beckers T, Kindt M (2017). Memory Reconsolidation Interference as an Emerging Treatment for Emotional Disorders: Strengths, Limitations, Challenges, and Opportunities. Annu Rev Clin Psychol 13: 99-121.

Ben Mamou C, Gamache K, Nader K (2006). NMDA receptors are critical for unleashing consolidated auditory fear memories. Nat Neurosci 9(10): 1237-1239.

Benchenane K, Tiesinga PH, Battaglia FP (2011). Oscillations in the prefrontal cortex: a gateway to memory and attention. Curr Opin Neurobiol 21(3): 475-485.

Bhatt DH, Zhang S, Gan WB (2009). Dendritic spine dynamics. Annu Rev Physiol 71: 261-282.

Bourne J, Harris KM (2007). Do thin spines learn to be mushroom spines that remember? Current opinion in neurobiology 17(3): 381-386.

Burgos-Robles A, Vidal-Gonzalez I, Quirk GJ (2009). Sustained conditioned responses in prelimbic prefrontal neurons are correlated with fear expression and extinction failure. J Neurosci 29(26): 8474-8482.

Cerqueira JJ, Taipa R, Uylings HB, Almeida OF, Sousa N (2007). Specific configuration of dendritic degeneration in pyramidal neurons of the medial prefrontal cortex induced by differing corticosteroid regimens. Cereb Cortex 17(9): 1998-2006.

Chen J, Dong B, Feng X, Jiang D, Chen G, Long C, et al (2020). Aberrant mPFC GABAergic synaptic transmission and fear behavior in neuroligin-2 R215H knock-in mice. Brain Res 1730: 146671.

Chen S, Tan Z, Xia W, Gomes CA, Zhang X, Zhou W, et al (2021). Theta oscillations synchronize human medial prefrontal cortex and amygdala during fear learning. Sci Adv 7(34).

Courtin J, Karalis N, Gonzalez-Campo C, Wurtz H, Herry C (2014). Persistence of amygdala gamma oscillations during extinction learning predicts spontaneous fear recovery. Neurobiol Learn Mem 113: 82-89.

Da Silva TR, Raymundi AM, Bertoglio LJ, Andreatini R, Stern CA (2020). Role of prelimbic cortex PKC and PKMzeta in fear memory reconsolidation and persistence following reactivation. Sci Rep 10(1): 4076. 
Da Silva WC, Cardoso G, Bonini JS, Benetti F, Izquierdo I (2013). Memory reconsolidation and its maintenance depend on L-voltage-dependent calcium channels and CaMKII functions regulating protein turnover in the hippocampus. Proc Natl Acad Sci U S A 110(16): 6566-6570.

Deinhardt K, Chao MV (2014). Shaping neurons: Long and short range effects of mature and proBDNF signalling upon neuronal structure. Neuropharmacology 76 Pt C: 603-609.

Eisenberg M, Kobilo T, Berman DE, Dudai Y (2003). Stability of retrieved memory: inverse correlation with trace dominance. Science 301(5636): 1102-1104.

Engert F, Bonhoeffer T (1999). Dendritic spine changes associated with hippocampal long-term synaptic plasticity. Nature 399(6731): 66-70.

Fenton GE, Halliday DM, Mason R, Bredy TW, Stevenson CW (2016). Sex differences in learned fear expression and extinction involve altered gamma oscillations in medial prefrontal cortex. Neurobiol Learn Mem 135: 66-72.

Finnie PS, Nader K (2012). The role of metaplasticity mechanisms in regulating memory destabilization and reconsolidation. Neurosci Biobehav Rev 36(7): 1667-1707.

Fukushima H, Zhang Y, Kida S (2021). Active Transition of Fear Memory Phase from Reconsolidation to Extinction through ERK-Mediated Prevention of Reconsolidation. J Neurosci 41(6): 1288-1300.

Gibon J, Buckley SM, Unsain N, Kaartinen V, Seguela P, Barker PA (2015). proBDNF and p75NTR Control Excitability and Persistent Firing of Cortical Pyramidal Neurons. J Neurosci 35(26): 9741-9753.

Gogolla N, Caroni P, Luthi A, Herry C (2009). Perineuronal nets protect fear memories from erasure. Science 325(5945): 1258-1261.

Hallock HL, Quillian HMt, Mai Y, Maynard KR, Hill JL, Martinowich K (2019). Manipulation of a genetically and spatially defined sub-population of BDNF-expressing neurons potentiates learned fear and decreases hippocampal-prefrontal synchrony in mice. Neuropsychopharmacology 44(13): 2239-2246.

Hill JL, Hardy NF, Jimenez DV, Maynard KR, Kardian AS, Pollock CJ, et al (2016). Loss of promoter IV-driven BDNF expression impacts oscillatory activity during sleep, sensory information processing and fear regulation. Transl Psychiatry 6(8): e873.

Holehonnur R, Phensy AJ, Kim LJ, Milivojevic M, Vuong D, Daison DK, et al (2016). Increasing the GluN2A/GluN2B Ratio in Neurons of the Mouse Basal and Lateral Amygdala Inhibits the Modification of an Existing Fear Memory Trace. J Neurosci 36(36): 9490-9504.

Hong I, Kim J, Lee S, Ko HG, Nader K, Kaang BK, et al (2013). AMPA receptor exchange underlies transient memory destabilization on retrieval. Proc Natl Acad Sci U S A 110(20): 8218-8223.

Jarome TJ, Ferrara NC, Kwapis JL, Helmstetter FJ (2016). CaMKII regulates proteasome phosphorylation and activity and promotes memory destabilization following retrieval. Neurobiol Learn Mem 128: 103-109.

Je HS, Yang F, Ji Y, Nagappan G, Hempstead BL, Lu B (2012). Role of pro-brain-derived neurotrophic factor (proBDNF) to mature BDNF conversion in activity-dependent competition at developing neuromuscular synapses. Proc Natl Acad Sci U S A 109(39): 15924-15929.

Kasai H, Fukuda M, Watanabe S, Hayashi-Takagi A, Noguchi J (2010). Structural dynamics of dendritic spines in memory and cognition. Trends Neurosci 33(3): 121-129.

Kasai H, Ziv NE, Okazaki H, Yagishita S, Toyoizumi T (2021). Spine dynamics in the brain, mental disorders and artificial neural networks. Nat Rev Neurosci 22(7): 407-422.

Kim-Cohen J, Caspi A, Moffitt TE, Harrington H, Milne BJ, Poulton R (2003). Prior juvenile 
diagnoses in adults with mental disorder: developmental follow-back of a prospective-longitudinal cohort. Arch Gen Psychiatry 60(7): 709-717.

Kim JH, Hamlin AS, Richardson R (2009). Fear extinction across development: the involvement of the medial prefrontal cortex as assessed by temporary inactivation and immunohistochemistry. J Neurosci 29(35): 10802-10808.

Kim JH, Li S, Richardson R (2011). Immunohistochemical analyses of long-term extinction of conditioned fear in adolescent rats. Cereb Cortex 21(3): 530-538.

Kim JH, Richardson R (2007). A developmental dissociation in reinstatement of an extinguished fear response in rats. Neurobiol Learn Mem 88(1): 48-57.

Kim JH, Richardson R (2008). The effect of temporary amygdala inactivation on extinction and reextinction of fear in the developing rat: unlearning as a potential mechanism for extinction early in development. J Neurosci 28(6): 1282-1290.

Kozisek ME, Middlemas D, Bylund DB (2008). The differential regulation of BDNF and TrkB levels in juvenile rats after four days of escitalopram and desipramine treatment. Neuropharmacology 54(2): 251-257.

Langlois A, Diabira D, Ferrand N, Porcher C, Gaiarsa JL (2013). NMDA-dependent switch of proBDNF actions on developing GABAergic synapses. Cereb Cortex 23(5): 1085-1096.

Langton JM, Kim JH, Nicholas J, Richardson R (2007). The effect of the NMDA receptor antagonist MK-801 on the acquisition and extinction of learned fear in the developing rat. Learn Mem 14(10): 665-668.

Lee JL (2009). Reconsolidation: maintaining memory relevance. Trends Neurosci 32(8): 413-420.

Li MY, Miao WY, Wu QZ, He SJ, Yan G, Yang Y, et al (2017). A Critical Role of Presynaptic Cadherin/Catenin/p140Cap Complexes in Stabilizing Spines and Functional Synapses in the Neocortex. Neuron 94(6): 1155-1172 e1158.

Li X, Sun W, An L (2018). Nano-CuO impairs spatial cognition associated with inhibiting hippocampal long-term potentiation via affecting glutamatergic neurotransmission in rats. Toxicol Ind Health 34(6): 409-421.

Liberman LC, Lipp OV, Spence SH, March S (2006). Evidence for retarded extinction of aversive learning in anxious children. Behav Res Ther 44(10): 1491-1502.

Likhtik E, Paz R (2015). Amygdala-prefrontal interactions in (mal)adaptive learning. Trends Neurosci 38(3): 158-166.

Liu XB, Murray KD, Jones EG (2004). Switching of NMDA receptor 2A and 2B subunits at thalamic and cortical synapses during early postnatal development. J Neurosci 24(40): 8885-8895.

Lundqvist M, Herman P, Lansner A (2011). Theta and gamma power increases and alpha/beta power decreases with memory load in an attractor network model. J Cogn Neurosci 23(10): 3008-3020.

Ma X, Vuyyuru H, Munsch T, Endres T, Lessmann V, Meis S (2021). ProBDNF Dependence of LTD and Fear Extinction Learning in the Amygdala of Adult Mice. Cereb Cortex.

Mattson MP, Maudsley S, Martin B (2004). BDNF and 5-HT: a dynamic duo in age-related neuronal plasticity and neurodegenerative disorders. Trends Neurosci 27(10): 589-594.

McCallum J, Kim JH, Richardson R (2010). Impaired extinction retention in adolescent rats: effects of D-cycloserine. Neuropsychopharmacology 35(10): 2134-2142.

Menshanov PN, Lanshakov DA, Dygalo NN (2015). proBDNF is a major product of bdnf gene expressed in the perinatal rat cortex. Physiol Res 64(6): 925-934.

Merikangas KR, He JP, Burstein M, Swanson SA, Avenevoli S, Cui L, et al (2010). Lifetime 
prevalence of mental disorders in U.S. adolescents: results from the National Comorbidity Survey Replication--Adolescent Supplement (NCS-A). J Am Acad Child Adolesc Psychiatry 49(10): 980-989. Merlo E, Milton AL, Everitt BJ (2018). A Novel Retrieval-Dependent Memory Process Revealed by the Arrest of ERK1/2 Activation in the Basolateral Amygdala. J Neurosci 38(13): 3199-3207.

Milad MR, Quirk GJ (2002). Neurons in medial prefrontal cortex signal memory for fear extinction. Nature 420(6911): 70-74.

Milton AL, Lee JL, Butler VJ, Gardner R, Everitt BJ (2008). Intra-amygdala and systemic antagonism of NMDA receptors prevents the reconsolidation of drug-associated memory and impairs subsequently both novel and previously acquired drug-seeking behaviors. J Neurosci 28(33): 8230-8237.

Milton AL, Merlo E, Ratano P, Gregory BL, Dumbreck JK, Everitt BJ (2013). Double dissociation of the requirement for GluN2B- and GluN2A-containing NMDA receptors in the destabilization and restabilization of a reconsolidating memory. J Neurosci 33(3): 1109-1115.

Mizoguchi Y, Ohgidani M, Haraguchi Y, Murakawa-Hirachi T, Kato TA, Monji A (2021). ProBDNF induces sustained elevation of intracellular $\mathrm{Ca}(2+)$ possibly mediated by TRPM7 channels in rodent microglial cells. Glia 69(7): 1694-1708.

Moench KM, Wellman CL (2017). Differential dendritic remodeling in prelimbic cortex of male and female rats during recovery from chronic stress. Neuroscience 357: 145-159.

Moriceau S, Sullivan RM (2006). Maternal presence serves as a switch between learning fear and attraction in infancy. Nat Neurosci 9(8): 1004-1006.

Mueller EM, Panitz C, Hermann C, Pizzagalli DA (2014). Prefrontal oscillations during recall of conditioned and extinguished fear in humans. J Neurosci 34(21): 7059-7066.

Nader K, Schafe GE, Le Doux JE (2000). Fear memories require protein synthesis in the amygdala for reconsolidation after retrieval. Nature 406(6797): 722-726.

Nyhus E, Curran T (2010). Functional role of gamma and theta oscillations in episodic memory. Neurosci Biobehav Rev 34(7): 1023-1035.

Ohno T, Maeda H, Murabe N, Kamiyama T, Yoshioka N, Mishina M, et al (2010). Specific involvement of postsynaptic GluN2B-containing NMDA receptors in the developmental elimination of corticospinal synapses. Proc Natl Acad Sci U S A 107(34): 15252-15257.

Orefice LL, Shih CC, Xu H, Waterhouse EG, Xu B (2016). Control of spine maturation and pruning through proBDNF synthesized and released in dendrites. Mol Cell Neurosci 71: 66-79.

Ozawa M, Davis P, Ni J, Maguire J, Papouin T, Reijmers L (2020). Experience-dependent resonance in amygdalo-cortical circuits supports fear memory retrieval following extinction. Nat Commun 11(1): 4358.

Paoletti P, Bellone C, Zhou Q (2013). NMDA receptor subunit diversity: impact on receptor properties, synaptic plasticity and disease. Nat Rev Neurosci 14(6): 383-400.

Pattwell SS, Bath KG, Casey BJ, Ninan I, Lee FS (2011). Selective early-acquired fear memories undergo temporary suppression during adolescence. Proc Natl Acad Sci U S A 108(3): 1182-1187.

Pattwell SS, Duhoux S, Hartley CA, Johnson DC, Jing D, Elliott MD, et al (2012). Altered fear learning across development in both mouse and human. Proc Natl Acad Sci U S A 109(40): 16318-16323.

Pattwell SS, Liston C, Jing D, Ninan I, Yang RR, Witztum J, et al (2016). Dynamic changes in neural circuitry during adolescence are associated with persistent attenuation of fear memories. Nat Commun 7: 11475.

Piantadosi PT, Floresco SB (2014). Prefrontal cortical GABA transmission modulates discrimination 
and latent inhibition of conditioned fear: relevance for schizophrenia. Neuropsychopharmacology 39(10): 2473-2484.

Radiske A, Gonzalez MC, Conde-Ocazionez S, Rossato JI, Kohler CA, Cammarota M (2020). Cross-Frequency Phase-Amplitude Coupling between Hippocampal Theta and Gamma Oscillations during Recall Destabilizes Memory and Renders It Susceptible to Reconsolidation Disruption. $J$ Neurosci 40(33): 6398-6408.

Riffault B, Kourdougli N, Dumon C, Ferrand N, Buhler E, Schaller F, et al (2018). Pro-Brain-Derived Neurotrophic Factor (proBDNF)-Mediated p75NTR Activation Promotes Depolarizing Actions of GABA and Increases Susceptibility to Epileptic Seizures. Cereb Cortex 28(2): 510-527.

Rosas-Vidal LE, Do-Monte FH, Sotres-Bayon F, Quirk GJ (2014). Hippocampal--prefrontal BDNF and memory for fear extinction. Neuropsychopharmacology 39(9): 2161-2169.

Roux F, Uhlhaas PJ (2014). Working memory and neural oscillations: alpha-gamma versus theta-gamma codes for distinct WM information? Trends Cogn Sci 18(1): 16-25.

Schiller D, Kanen JW, LeDoux JE, Monfils MH, Phelps EA (2013). Extinction during reconsolidation of threat memory diminishes prefrontal cortex involvement. Proc Natl Acad Sci U S A 110(50): 20040-20045.

Sheng M, Cummings J, Roldan LA, Jan YN, Jan LY (1994). Changing subunit composition of heteromeric NMDA receptors during development of rat cortex. Nature 368(6467): 144-147.

Sotres-Bayon F, Diaz-Mataix L, Bush DE, LeDoux JE (2009). Dissociable roles for the ventromedial prefrontal cortex and amygdala in fear extinction: NR2B contribution. Cereb Cortex 19(2): 474-482.

Sotres-Bayon F, Sierra-Mercado D, Pardilla-Delgado E, Quirk GJ (2012). Gating of fear in prelimbic cortex by hippocampal and amygdala inputs. Neuron 76(4): 804-812.

Stark E, Eichler R, Roux L, Fujisawa S, Rotstein HG, Buzsaki G (2013). Inhibition-induced theta resonance in cortical circuits. Neuron 80(5): 1263-1276.

Sun Q, Li X, Li A, Zhang J, Ding Z, Gong H, et al (2020a). Ventral Hippocampal-Prefrontal Interaction Affects Social Behavior via Parvalbumin Positive Neurons in the Medial Prefrontal Cortex. iScience 23(3): 100894.

Sun W, Che H, Li J, Tang D, Liu X, Liu W, et al (2020b). Dorsolateral Striatal proBDNF Improves Reversal Learning by Enhancing Coordination of Neural Activity in Rats. Mol Neurobiol 57(11): 4642-4656.

Sun W, Cheng H, Yang Y, Tang D, Li X, An L (2021a). Requirements of Postnatal proBDNF in the Hippocampus for Spatial Memory Consolidation and Neural Function. Front Cell Dev Biol 9: 678182.

Sun W, Li J, Cui S, Luo L, Huang P, Tang C, et al (2019). Sleep Deprivation Disrupts Acquisition of Contextual Fear Extinction by Affecting Circadian Oscillation of Hippocampal-Infralimbic proBDNF. eNeuro 6(5).

Sun W, Li X, An L (2018a). Distinct roles of prelimbic and infralimbic proBDNF in extinction of conditioned fear. Neuropharmacology 131: 11-19.

Sun W, Li X, Tang C, An L (2018b). Acute Low Alcohol Disrupts Hippocampus-Striatum Neural Correlate of Learning Strategy by Inhibition of PKA/CREB Pathway in Rats. Front Pharmacol 9: 1439.

Sun W, Tang D, Yang Y, Wu Z, Li X, An L (2021b). Melamine impairs working memory and reduces prefrontal activity associated with inhibition of AMPA receptor GluR2/3 subunit expression. Toxicol Lett 350: 171-184.

Sun W, Wu Y, Tang D, Li X, An L (2021c). Melamine disrupts spatial reversal learning and learning 
strategy via inhibiting hippocampal BDNF-mediated neural activity. PLoS One 16(1): e0245326.

Sun W, Yang Y, Chen X, Cheng Y, Li X, An L (2021d). Light Promotes Neural Correlates of Fear Memory via Enhancing Brain-Derived Neurotrophic Factor (BDNF) Expression in the Prelimbic Cortex. ACS Chem Neurosci 12(10): 1802-1810.

Sun W, Yang Y, Wu Z, Chen X, Li W, An L (2021e). Chronic Cyanuric Acid Exposure Depresses Hippocampal LTP but Does Not Disrupt Spatial Learning or Memory in the Morris Water Maze. Neurotox Res 39(4): 1148-1159.

Takita M, Fujiwara SE, Izaki Y (2013). Functional structure of the intermediate and ventral hippocampo-prefrontal pathway in the prefrontal convergent system. J Physiol Paris 107(6): 441-447.

Thomases DR, Cass DK, Meyer JD, Caballero A, Tseng KY (2014). Early adolescent MK-801 exposure impairs the maturation of ventral hippocampal control of basolateral amygdala drive in the adult prefrontal cortex. J Neurosci 34(27): 9059-9066.

Tort AB, Kramer MA, Thorn C, Gibson DJ, Kubota Y, Graybiel AM, et al (2008). Dynamic cross-frequency couplings of local field potential oscillations in rat striatum and hippocampus during performance of a T-maze task. Proc Natl Acad Sci U S A 105(51): 20517-20522.

Tronson NC, Taylor JR (2007). Molecular mechanisms of memory reconsolidation. Nat Rev Neurosci 8(4): 262-275.

Voloh B, Valiante TA, Everling S, Womelsdorf T (2015). Theta-gamma coordination between anterior cingulate and prefrontal cortex indexes correct attention shifts. Proc Natl Acad Sci U S A 112(27): 8457-8462.

von Bohlen und Halbach O (2010). Involvement of BDNF in age-dependent alterations in the hippocampus. Front Aging Neurosci 2.

Wang SH, de Oliveira Alvares L, Nader K (2009). Cellular and systems mechanisms of memory strength as a constraint on auditory fear reconsolidation. Nat Neurosci 12(7): 905-912.

Weber JP, Andrasfalvy BK, Polito M, Mago A, Ujfalussy BB, Makara JK (2016). Location-dependent synaptic plasticity rules by dendritic spine cooperativity. Nat Commun 7: 11380.

Woo NH, Teng HK, Siao CJ, Chiaruttini C, Pang PT, Milner TA, et al (2005). Activation of p75NTR by proBDNF facilitates hippocampal long-term depression. Nat Neurosci 8(8): 1069-1077.

Xu ZQ, Sun Y, Li HY, Lim Y, Zhong JH, Zhou XF (2011). Endogenous proBDNF is a negative regulator of migration of cerebellar granule cells in neonatal mice. Eur J Neurosci 33(8): 1376-1384.

Yang F, Je HS, Ji Y, Nagappan G, Hempstead B, Lu B (2009a). Pro-BDNF-induced synaptic depression and retraction at developing neuromuscular synapses. J Cell Biol 185(4): 727-741.

Yang G, Pan F, Gan WB (2009b). Stably maintained dendritic spines are associated with lifelong memories. Nature 462(7275): 920-924.

Yang J, Harte-Hargrove LC, Siao CJ, Marinic T, Clarke R, Ma Q, et al (2014). proBDNF negatively regulates neuronal remodeling, synaptic transmission, and synaptic plasticity in hippocampus. Cell Rep 7(3): 796-806.

Yang J, Siao CJ, Nagappan G, Marinic T, Jing D, McGrath K, et al (2009c). Neuronal release of proBDNF. Nat Neurosci 12(2): 113-115.

Yasuda H, Mukai H (2015). Turning off of GluN2B subunits and turning on of CICR in hippocampal LTD induction after developmental GluN2 subunit switch. Hippocampus 25(11): 1274-1284.

Zhang JJ, Haubrich J, Bernabo M, Finnie PSB, Nader K (2018). Limits on lability: Boundaries of reconsolidation and the relationship to metaplasticity. Neurobiol Learn Mem 154: 78-86. 


\section{Figure Legends}

Figure 1. The higher prelimbic proBDNF expression during the juvenile period facilitates retrieval-dependent memory destabilization.

Quantification of the proBDNF (A) and its receptor $\mathrm{p} 75^{\mathrm{NTR}}$ (B) levels in prelimbic cortex of juvenile and adult rats. Representative immunoblots the expression of proBDNF and $\mathrm{p} 75^{\mathrm{NTR}}$ (Top). A significant increase in the proBDNF levels was detected in juvenile group, as well the $\mathrm{p} 75^{\mathrm{NTR}}$ levels (Bottom). $(* P<0.05)$. (C) Both juvenile and adult rats conditioned with eight tones froze significantly more than rats trained with four tones during the LTM test. $(* P<0.05$, four-tone group vs. eight-tone group). (D) Schematic describing the behavioral timeline for the retrieval-dependent memory destabilization experiment using rats conditioned with four tones (left). Rats were auditory fear conditioned with four tones. Retrieval of the fear memory was induced by exposing the rats to a single tone presentation five days following the fear condition learning, and then the rats were immediately infused with proBDNF, its antibody, anisomycin or vehicle into the prelimbic cortex. Two days later, the rats were exposed to tones in a novel context during the PR-LTM test and freezing was measured. No significant difference in the average percentage freezing across four tones during the memory retrieval but the percentage freezing during the PR-LTM test was significant lower in juvenile group than adult group (Right). Infusions of anti-proBDNF antibody obviously enhanced the freeze behavior of juvenile but not adult rats. Microinjections of anisomycin and proBDNF into the prelimbic region could enhance the freezing levels of juvenile rats. $(* P<0.05$, Juvenile group vs. other groups; $\# P<0.05$, Juvenile+Pro or Juvenile+Anis group vs. other groups). (E) Schematic describing the behavioral timeline for the retrieval-dependent memory destabilization experiment using rats conditioned with eight tones (left). No significant difference in either the average percentage freezing across eight tones during the memory retrieval or the average percentage freezing during the PR-LTM test, which was conducted two days later (Right). (F) Schematic describing the experimental timeline for a reconsolidation experiment using rats conditioned with four tones and a PR-LTM test conducted 30 min later (left). No significant difference in either the average percentage freezing across four tones during the memory 
retrieval or the average percentage freezing during the PR-LTM test (Right). (G) Schematic describing the experimental timeline for a reconsolidation experiment using rats conditioned with four tones and drugs were infused one day after the memory retrieval (left). No significant difference in either the average percentage freezing across four tones during the memory retrieval but infusions of anisomycin and proBDNF did not enhance the freeze behavior during the PR-LTM test, which was performed one day after the microinfusions (Right). (H) Schematic describing the experimental timeline for fear extinction and reinstatement using rats conditioned with four tones (left). Rats were fear conditioned with four tones, and five days later, fear extinction learning was performed. The extinguished fear was reinstated one day following memory extinction. Juvenile group was indistinguishable from adult group in the acquisition of fear extinction training (Right). (I) Schematic describing the experimental timeline for fear expression (left). For tone reactions and the expression of fear behavior, the mean freezing levels during the first three tones of the extinction training were calculated. There was no significant difference in the percentage freezing (Right). The number of rats in each group used was indicated in each column/figure.

\section{Figure 2. Up-regulation of proBDNF-p75NTR signaling mediated by} NMDA-GluN2B contributes to enhance the modulation of existing fear memory traces in juvenile rats.

(A) Schematic describing the behavioral timeline for the retrieval-dependent memory destabilization experiment using rats conditioned with four tones (Top). Immediately following the memory retrieval, the rats infused with TAT-Pep5, K252a or vehicle into the prelimbic cortex 15 min prior to the mBDNF, proBDNF or vehicle infusion. Two days later, PR-LTM was assessed by exposed the rats to the novel context. Similar, no significant difference in the percentage freezing during the memory retrieval but the percentage freezing level during the PR-LTM test was significant lower in juvenile group than adult group (Bottom). No obvious effect of mBDNF on freeze behavior was found. Infusions of $\mathrm{p} 75^{\mathrm{NTR}}$ blocker TAT-Pep5 could significantly enhance the percentage of freeze behavior. Meanwhile, infusions of TAT-Pep5, but 
not K252a, markedly blocked the effects of proBDNF treatment. $(* P<0.05)$. Representative immunoblots the expression of the expression of the NMDA-GluN2A (B) and NMDA-GluN2B (C) receptors in prelimbic cortex (Top). The level of GluN2A subunit was significantly higher but the level of GluN2B subunit was significantly lower compared adult group with juvenile group. $(* P<0.05)$. (C) Schematic describing the behavioral timeline for the retrieval-dependent memory destabilization experiment using rats conditioned with four tones (Top). Immediately following the memory retrieval, the rats infused with CPP, NVP-AAM077, Ro25-6981 or vehicle into the prelimbic region $15 \mathrm{~min}$ prior to the proBDNF or vehicle infusion. Two days later, the PR-LTM was tested as above. Both the broad NMDA receptor inhibitor $\mathrm{CPP}$ and The NMDA-GluN2B subunit antagonist Ro25-6981 enhanced the percentage freeze level during the PR-LTM test. However, the NVP-infused rats could maintain the lower percentage freeze level. Furthermore, blockage of the NMDA-GluN2B subunit could completely block the effects of proBDNF on freeze behavior. The number of rats in each group used was indicated in each column/figure.

Figure 3. The increase proBDNF alters synaptic currents, promotes LFS-induced synaptic depression and strengthens the theta phase-gamma amplitude coupling during the PR-LTM test.

(A) Schematic describing the timeline for morphological analysis (Top-left). Illustration of the region of interest in prelimbic images (Top-middle), and dendritic segment analysis for spine quantification (Top-right). Red circles indicated the mushroom type spine, yellow circles indicated thin type spine and blue circles indicated stubby type spine. Sample images were projected at minimal intensity and inverted, background was then subtracted, followed by brightness/contrast adjustment. Scale bars, $5 \mu \mathrm{m}$. Quantification of spine density (Bottom-left) and the proportion of spine (Bottom-right). No statistical difference in spine density was found between juvenile and adult groups. However, a significant higher proportion of thin type spine but a lower mushroom type spine was observed in juveniles compared with adults. (B) Schematic describing the timeline for EPSCs recordings (Top-left). Representative 
continuous traces (Top-middle) and average waveform (Top-right) of the pharmacologically isolated NMDA EPSCs in the prelimbic neurons of adult, juvenile and juvenile+anti groups. No change in the amplitude of EPSCs (Bottom-left) was found but the frequency (Bottom-middle) and decay time (Bottom-right) were significantly increased in juvenile group. The enhanced frequency and decay time of NMDA currents in juvenile group were inhibited after infusions of anti-proBDNF antibody. ( ${ }^{*} P<0.05$, Adult or Juvenile+Anti group vs. Juvenile group). (C) Schematic describing the timeline for the LFS-induced LTD recordings (Top-left). Schematic representative placements of the stimulation electrodes (vHPC; ventral hippocampus) and recording electrodes (mPFC) (Top-middle). Numbers in figures were referenced to bregma. The black traces indicated the baseline traces and the dark gray ones indicated the traces of the fEPSPs, which were taken at 41 min after LFS (Top-right). Characteristic time courses of fEPSP slope (Bottom-left). Bidirectional arrow represented application of LFS (300 pulses at $1.0 \mathrm{~Hz}$ ). Time coursing changes in fEPSPs slope. Magnitude of LTD was determined as responses in the last $20 \mathrm{~min}$ (between 41 and 60 min following the LFS). The LFS could induce a stabilized LTD in juvenile group but not adult group. This form of LTD could be drastically inhibited by GluN2B antagonist Ro25-6981 but not GluN2A antagonist NVP-AAM077. ( ${ }^{*} P<0.05$, Juvenile or Juvenile+NVP group vs. other groups). (D) Example of filtered prelimbic LFP recordings of juvenile, adult and juvenile+anti animals during the PR-LTM test. Black lines, LFP filtered in the thta frequency range $(8-12 \mathrm{~Hz})$; gray line, LFP filtered in the gamma frequency range $(30-100 \mathrm{~Hz})$. Representative phase-amplitude diagrams (Top-right). The color scale represents the values of mean modulation index (MI). Juvenile rats showed a higher MI value than adult group while block of prelimbic proBDNF by its antibody dramatically reduced the MI value of juvenile group (Bottom-left). There was no statistical difference in the mean power of theta (Bottom-middle) or gamma (Bottom-right) oscillations. $(* P<0.05$, Juvenile or Juvenile+NVP group vs. other groups). The number of rats in each group used was indicated in each column/figure. 
(A) ${ }_{\beta \text {-actin }}^{\text {proBDNF }}$

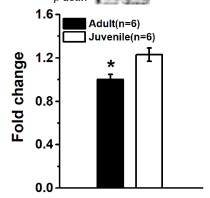

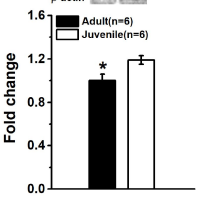
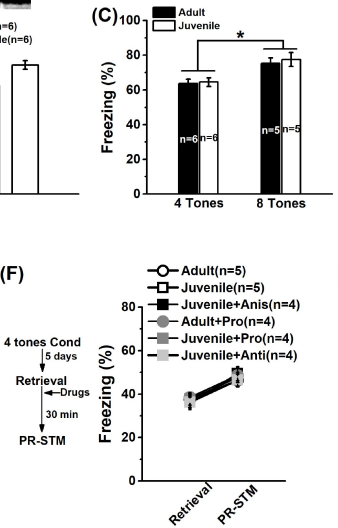

(G)

4 tones Cond

5 days

Retrieval

Retrieval

PR-LTM

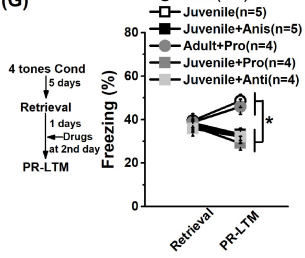

(F)

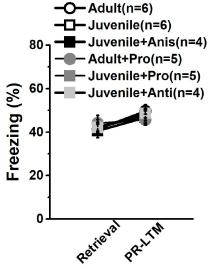

(H)

(E)

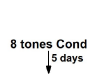
Retrieval

2 days

PR-LTM

P

4 tones Cond

Extinction

Reinstatment

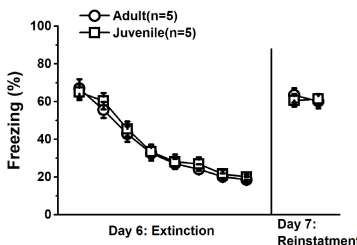

(I)

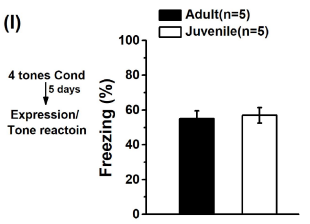


(A) 4 tones Cond

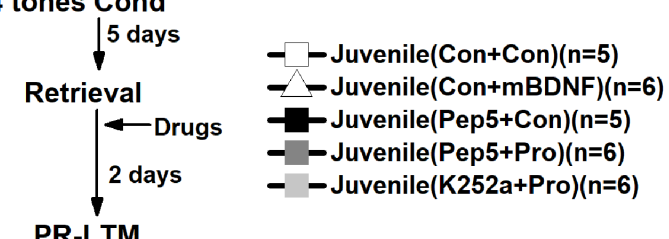

PR-LTM

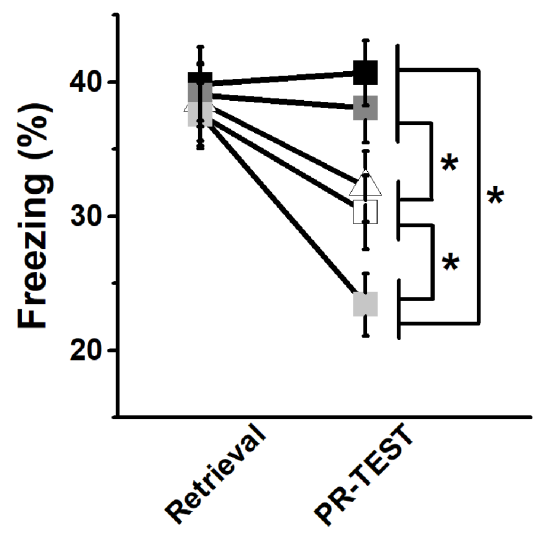

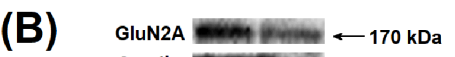
$\beta$-actin

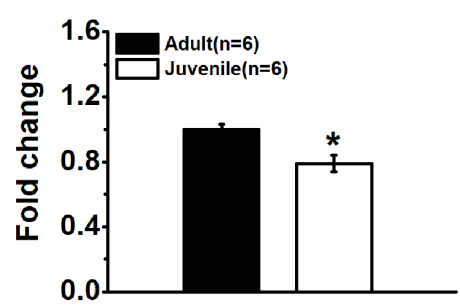

(C) GluN2B Easo eass $\longleftarrow 180 \mathrm{kDa}$

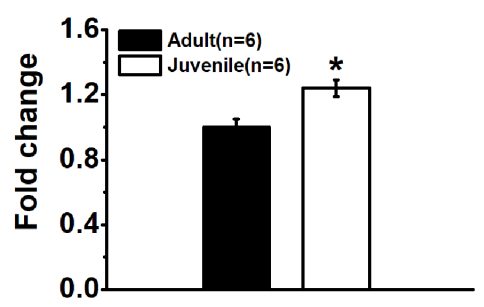

(D) 4 tones Cond

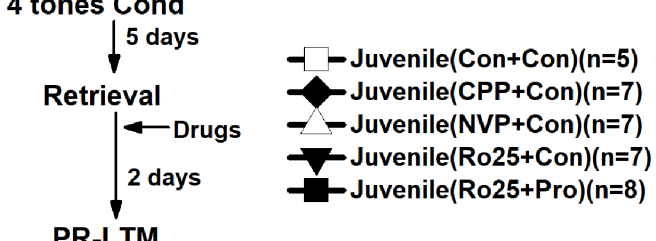

PR-LTM

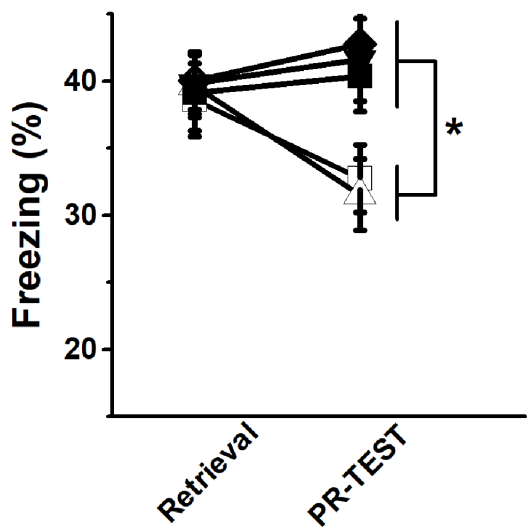



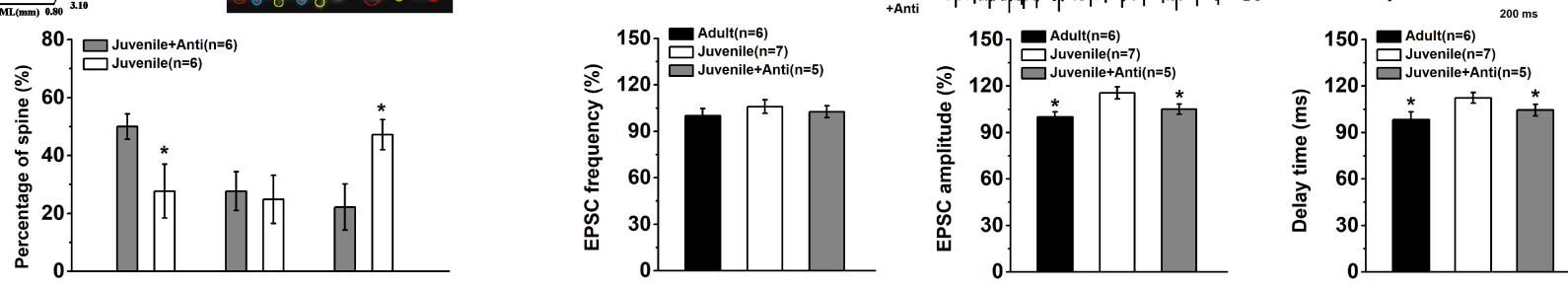

(C

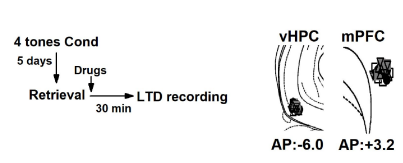

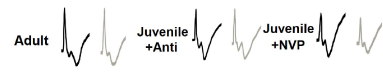

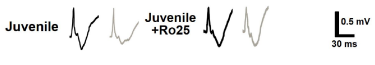

(D)

Adult Amwinuminuming $1200 \mathrm{Nu}$

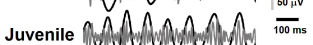

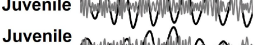

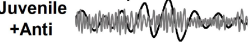
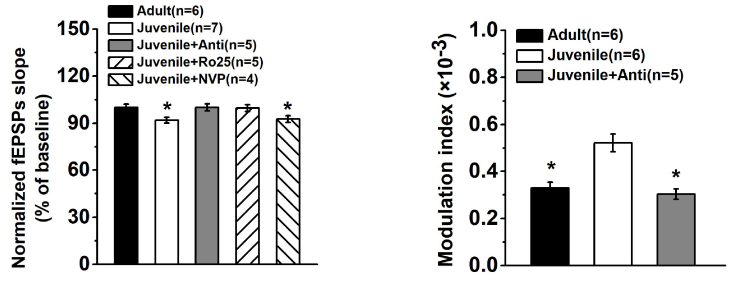
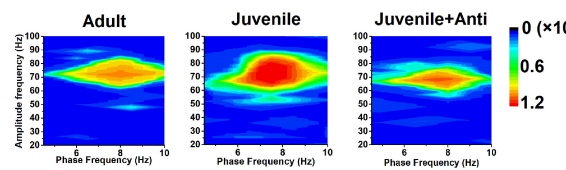
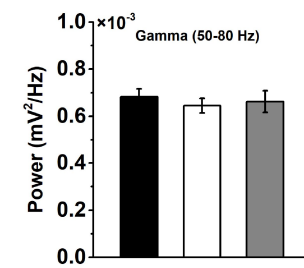